\title{
INDICADORES DE AVALIAÇÃO DE POLÍTICAS PÚBLICAS PARA EAD
}

\author{
MOSSORÓ/RN JUNHO/2018
}

\author{
Kátia Cilene da Silva - UFERSA - katiacs@ufersa.edu.br \\ Ana Beatriz Gomes Pimenta de Carvalho - UFPE - anabeatrizgpc@gmail.com \\ Querte Terezinha Conzi Mehlecke - FACCAT - quertecm@gmail.com \\ Tipo: Investigação Científica (IC) \\ Natureza: Descrição de Projeto em Andamento \\ Categoria: Estratégias e Políticas \\ Setor Educacional: EDUCAÇÃO SUPERIOR
}

\begin{abstract}
RESUMO
O presente artigo faz parte de uma pesquisa mais abrangente para uma tese de doutorado que se propõe a apresentar um modelo de avaliação para a Universidade Aberta do Brasil (UAB) como política pública para educação à distância. O presente recorte tem por objetivo apresentar as dimensões e as variáveis de análise adotadas para o desenvolvimento do modelo de avaliação proposto, bem como as referências formais que foram utilizadas como fontes de pesquisa. Para tanto foi realizada uma extensa revisão de literatura sobre a área, através da qual foram identificados os indicares de avaliação utilizados para as diferentes dimensões de análise; sendo que, para as dimensões não contempladas pelos estudos pesquisados, os indicadores foram criados pela pesquisadora, com base em sua experiência com o sistema UAB. Como resultado da presente pesquisa tem-se os possíveis valores que cada variável pode assumir quando da aplicação do modelo para avaliação do Sistema UAB.
\end{abstract}

Palavras-chave: Avaliação de políticas públicas; Políticas públicas para EaD; Dimensões de avaliação; Variáveis de análise; Métricas de avaliação. 


\section{INTRODUÇÃO}

O atual contexto de desregulamentação e de desacionamento do estado do bem-estar social influiu para que as Instituições de Ensino Superior (IES) públicas não acompanhassem o crescimento das demandas sociais por serviços de educação e formação profissional, uma vez que as IES privadas respondem por mais de $77,11 \%$ do número das IES (TRIGUEIRO, 2000). As políticas de ajuste econômico e de contenção de despesas afetaram o crescimento das IES públicas, ocasionando déficit educacional. As reformas educacionais têm pressionado as IES públicas através da exigência de expansão de matrículas, redução da duração na carga horária de diversos cursos, criação de cursos noturnos e estabelecimento de redes e consórcios de Educação a Distância $(\mathrm{EaD})$.

Para Ferrugini, Morais, Pereira e Souza (2013) a EaD se apresenta como um relevante instrumento da Política Pública de expansão da Ensino Superior (ES), em função das desigualdades ainda existentes do país e do potencial da EaD em minimizá-las, principalmente no que se refere à democratização do acesso à educação. Através dela busca-se reverter esse quadro de desigualdade proporcionando a democratização do acesso através da expansão e da interiorização do ES. Zuin (2006) afirma que, neste contexto, o Sistema Universidade Aberta do Brasil (UAB) prioriza os cursos de formação superior para professores das redes públicas de ensino básico, e para formação em administração pública, assim atendendo aos dois públicos: a) docentes; e, b) gestores. Neste contexto surge a necessidade de apresentar um modelo de avaliação que mensure a efetividade da UAB como política pública de EaD, utilizada como estratégia para expansão do ensino superior no país.

\section{FUNDAMENTAÇÃO TEÓRICA}

Para composição das variáveis e suas respectivas categorias de análise adotadas para análise dos aspectos relacionados à "caracterização do sistema UAB" foram utilizadas referências formais da área que versam sobre cada um dos aspectos a serem analisados. Para 0 aspecto Contribuição da EaD como política pública educacional foram utilizadas as referências de contribuição previstas pelo próprio (MEC, 2016). Já para o aspecto Relevância das políticas públicas para a EaD no Brasil foram utilizadas as referências da própria UAB, apresentadas pela CAPES (CAPES, 2016a), enquanto que para o aspecto Alcance dos objetivos da UAB foram utilizados os descritos no Decreto Presidencial no 5.800 (BRASIL, 2006a). Finalizando a composição do grupo de aspectos constitutivos da UAB a Consideração dos eixos fundamentais de sustentação da UAB foi fundamentada nos eixos apresentados pela 
CAPES (CAPES, 2016b).

Quanto aos aspectos vinculados ao relacionamento dos atores do sistema o único baseado no referencial foi o da Colaboração, para o qual foram propostas as categorias de análise apresentadas por (REUSE, 2013). Os demais aspectos como Interação ente os participantes do sistema, Preparação dos coordenadores para suas atribuições e Gestão do polo (dificuldade de adaptação da gestão do polo à diversidade de IPES nele ofertantes de cursos) foram propostos pela pesquisadora, bem como suas categorias de análise foram elaboradas pela mesma com base em sua experiência de atuação na área. Já o aspecto Dificuldades na gestão da UAB foi uma proposição de adaptação pela pesquisadora do apresentado em Mill e Carmo (2012). Por se caracterizar como um aspecto fundamental para a efetividade das políticas educacionais para EaD a institucionalização foi tratada no aspecto Estágio de institucionalização, para o qual foram utilizadas as categorias de análise propostas por Ferreira e Carneiro (2015), em seus artigo sobre a análise do Sistema UAB. Outro aspecto considera extremamente relevante para a manutenção da flexibilidade das ações no Sistema UAB é o de Melhores práticas compartilhadas, cuja proposição foi baseada em UAB/UFES/IFES (2013), com adaptações da pesquisadora. Já o aspecto relacionado a Autonomia dos atores do sistema foi baseado nos estudos de Melo e Silva (1991) sobre a gestão e a autonomia da escola nas novas propostas de políticas educativas para a América Latina.

Para composição das variáveis e suas respectivas categorias de análise adotadas para análise dos aspectos relacionados ao "diagnóstico do sistema UAB" foram utilizadas referências formais da área que versam sobre cada um dos aspectos a serem analisados. Para o aspecto Impactos no ensino superior foram utilizadas as categorias propostas por Mercado et al. (2012) para avaliação dos impactos da UAB. Já para o aspecto Desafios foram utilizadas as categorias propostas por Sarturi et al. (2015) em combinação com as identificadas por Lira e Lima (2014), enquanto que para o aspecto Fatores críticos foram utilizadas as categorias propostas por Michelon e Razuck (2016) em seu estudo sobre os fatores críticos da gestão integrada na UAB.

\section{METODOLOGIA}

Para o desenvolvimento da pesquisa e validação das dimensões, categorias e variáveis de análise propostas para composição do modelo de avaliação da política pública foram aplicados questionários de coleta de dados, com o objetivo de verificar a efetiva ocorrência dos aspectos elencados para análise. Na construção dos instrumentos de coleta foram considerados alguns aspectos, como (NOGUEIRA, 2002, p. 1): a) objetivos 
do questionário; b) tipos de questionários; c) sequência das questões. Quanto aos objetivos, pode-se dizer que o questionário possui os dois objetivos possíveis, segundo Perrien (1986, p. 57): a) posicionamento demográfico (idade, grau de escolaridade, atividade, renda, etc...); e, b) estilo de vida (traduzido sob os aspectos de atitudes, interesses e opiniões).

No que se refere aos tipos de questionários, estes são classificados utilizando-se critérios como (NOGUEIRA, 2002, p. 1): a) quanto à relação entre subjetividade $X$ objetividade das questões - podem ser abertos ou fechados; b) quanto à forma de resposta - podem ser diretos ou indiretos; e, c) quanto à forma de aplicação - podem ser assistidos ou não assistidos. Quanto à relação entre subjetividade X objetividade das questões, optou-se pela elaboração de um questionário fechado, para o qual as variáveis e categorias de análise precisaram ser pensadas a priori, possibilitando maior agilidade na análise dos dados e permitindo tanto a análise de conteúdos (qualitativa) a partir das categorias previamente definidas, quanto a análise estatística (quantitativa); segundo as características postuladas por Nogueira (2002, p. 1):

Os questionários fechados, que apesar de se apresentarem de forma mais rígida do que os abertos, permite a aplicação direta de tratamentos estatísticos com auxílio de computadores e elimina a necessidade de se classificar respostas à posteriori, possivelmente induzindo tendências indesejáveis (NOGUEIRA, 2002, p. 1).

Quanto à forma de resposta, optou-se pela elaboração de um questionário indireto, dada a dificuldade de tabulação dos questionários com respostas abertas e a possibilidade da não obtenção de resultados muito precisos, o que é imprescindível para um processo avaliativo. Para tanto, foram pensadas previstas as alternativas a serem disponibilizadas aos respondentes, quando afirma que "os questionários indiretos, alternativas utilizadas para os casos em que não é possível obter uma resposta precisa às questões por impossibilidade ou por se tratar de um assunto delicado", para algumas das questões foi proposta uma alternativa aberta onde o respondente pode acrescentar uma ou mais alternativas que não tenham sido previstas à priori. Já no que se refere à forma de aplicação optou-se por questionários não assistidos, em função da decisão metodológica de realizar a coleta de dados dos questionários de forma online; decisão esta que, segundo Nogueira (2002, p. 1), pode ser considerado uma vantagem, pois:

Os questionários não assistidos, que se por um lado eliminam a possibilidade de contaminação por parte de um aplicador, podem não ser respondidos ou respondidos por pessoas não adequadas (NOGUEIRA, 2002, p. 1).

Então, o questionário fechado, indireto e não assistido elaborado para a presente pesquisa foi organizado em 4 seções, a saber: a) a IPES; b) o gestor da EaD; c) a caracterização do sistema UAB; e, d) o diagnóstico do sistema UAB. A seção "a IPES" 
possui questões que possibilitarão a identificação do perfil da instituição cujo coordenador foi sujeito da pesquisa. A seção "o gestor da EaD" possui questões que possibilitarão a identificação do perfil do coordenador, tanto no que diz respeito ao perfil social, como aos perfis profissional e de formação. Já a seção "a caracterização do sistema UAB" possui questões que possibilitarão a compreensão da contribuição e relevância da $\mathrm{EaD}$, os objetivos e eixos fundamentais da $U A B$, níveis de colaboração, interação, autonomia e preparação dos atores componentes do sistema UAB. Enquanto que a seção "o diagnóstico do sistema UAB" possui questões que possibilitarão a identificação dos impactos, desafios e fatores críticos relacionados ao alcance dos resultados pretendidos. O terceiro aspecto importante na elaboração de instrumentos de colega indicado por Nogueira (2002, p. 1) é a sequência das questões, que devem estar dispostas em uma sequência lógica que facilite a compreensão por parte do respondente, como sugerido por Perrien (1986, p. 63) sugere que:

As questões iniciais abordem temas abertos, de fácil resposta, com o objetivo primordial de envolver o respondente; As questões mais importantes e delicadas sejam inseridas no meio do questionário (PERRIEN,1986, p. 63).

Neste contexto, os dois roteiros de entrevistas, tanto para responsáveis na Diretoria de Educação a Distância (DED), quanto para membros da presidência do fórum de coordenadores foram organizados nas mesmas 4 seções adotadas para a organização e sequenciamento lógico das perguntas do questionário. Pelas 4 seções utilizadas para organizar tanto o questionário quanto os roteiros de entrevistas perpassam as dimensões que serão adotadas para a análise dos dados, bem como as variáveis componentes de cada dimensão. Outros cuidados tomados durante a construção do questionário foram os sugeridos por Miller (1977), os quais dizem respeito à disponibilizar aos respondentes todas as alternativas possíveis para resposta à uma questão e apresentar somente uma ideia em cada item de resposta.

\subsection{Dimensões de análise}

As dimensões de análise foram aqui adotadas com o objetivo de serem os parâmetros utilizados para descrever os fenômenos observados. Foram utilizados os conceitos de dimensões da política postulados por Frey (2000, p. 215), a saber: a) dimensão institucional; b) dimensão processual; e, c) dimensão material. A dimensão institucional trata das capacidades organizacionais, pois compreende os ativos ou recursos tangíveis (recursos humanos, marco legal e regulatório e estrutura física e financeira) e intangíveis (arranjos institucionais, questões comportamentais e comunicacionais, entre outros) que compõem a agenda proposta pela política a ser avaliada. Já a dimensão processual trata dos processos institucionais, considerando as estruturas organizacionais, as 
estratégias ou os procedimentos por meio dos quais as políticas são implementadas. Enquanto que a dimensão material trata das entregas de bens e serviços à sociedade, pois compreende os produtos físicos, monetários, normativos ou institucionais, efetivamente entregues à sociedade como resultados da política pública.

Como contribuição para a análise dos dados a dimensão institucional contribui para a realização de avaliações de formulação e desenho, enquanto que a processual subsidia o monitoramento e a avaliação de implementação e gestão e a material permite o monitoramento e a avaliação de eficiência.

\subsection{Variáveis de análise}

Para composição do questionário foram adotadas diferentes medidas para as escalas a utilizadas, em função das diferentes variáveis a serem observadas. Sendo assim foi necessária a adoção de 3 dos quatro níveis existentes de mensuração das escalas, a saber: a) nível nominal; b) nível ordinal; e, c) nível intervalar. Tais níveis são assim descritos por Aaker e Day (1990, p 42):

O nível nominal, que apenas identifica um determinado elemento. Não há nenhuma indicação de ordem ou comparação com outros elementos; O nível ordinal, no qual os elementos são ordenados, trazendo informações tais como o elemento A é maior do que o B, que por sua vez é menor do que C. - O nível intervalar, que apresenta, além da característica da ordenação, a de cada ponto na escala apresentar igual distância de seus pontos adjacentes, isto é, a distância entre os pontos 2 e 3 é igual a entre os pontos 5 e 6, por exemplo (AAKER e $D A Y, 1990, p$ 42).

Para melhor organização das variáveis de análise estas serão aqui apresentadas de acordo com a seção na qual aparecem nos instrumentos de coleta de dados e contemplam as quatro grandes classes de variáveis apresentadas por Nogueira (2002, p 4), a saber:

\footnotetext{
Atributos - que são as características pessoais ou demográficas (...) Comportamentais - que correspondem as características do comportamento do elemento (..) Conhecimento (beliefs) - que capturam as crenças do elemento pesquisado, que podem estar de acordo com a realidade dos fatos ou não (...) Atitudes - são as variáveis que captam o processo de avaliação e julgamento do entrevistado, em seu processo de ação (...) (NOGUEIRA, 2002, $p$ 4).
}

Também foram pensados os diferentes valores que cada variável pode assumir, os quais foram aqui dispostos em quadros descritivos e organizados segundo a seção do questionário que compõe.

\section{DISCUSSÃO DOS RESULTADOS}


Na dimensão "A IPES" são utilizadas variáveis como a esfera administrativa, a organização acadêmica, o Estado sede, a região do Estado na qual a sede se encontra, o tempo de existência e o tempo de credenciamento da instituição para oferta da EaD. $\mathrm{Na}$ dimensão "o gestor da EaD" são utilizadas variáveis como faixa etária, seco, maior titulação, área do curso de graduação, área do curso de mestrado, área do curso de doutorado, experiência docente na educação básica, tempo de experiência na educação básica, ocupou cargo de direção na educação básica, tempo que ocupou cargo de direção na educação básica, tempo de experiência no ensino superior, experiência em $\mathrm{EaD}$ anterior ao cargo de coordenador, tipo de experiência anterior com EaD, tempo total de experiência com EaD, tempo de trabalho na IPES, regime de trabalho na IPES, função que desempenha na $U A B$, tempo que exerce a função atual, possui formação específica em EaD e tipo de formação específica em EaD.

$\mathrm{Na}$ dimensão "a caracterização do sistema UAB" as variáveis estão organizadas em categorias, as saber: a) percepção da contribuição da EaD como política pública educacional; b) relevância das políticas públicas para a EaD no Brasil; c) alcance dos objetivos da UAB; d) consideração dos eixos fundamentais de sustentação da UAB; e) colaboração com variáveis como nível de colaboração entre a CAPES e a IPES, entre a IPES e os polos, entre a IPES e as prefeituras, no sistema UAB; f) interação entre os participantes do sistema; g) preparação dos coordenadores institucionais da UAB para realização de suas atribuições; h) gestão do polo, com a variável preparação para lidar com a diversidade de IPES; I) dificuldades na gestão da UAB; j) estágio de institucionalização do planejamento da EaD; k) estágio da institucionalização; I) estágio de institucionalização da infraestrutura para EaD; $\mathrm{m}$ ) estágio de institucionalização da gestão de pessoas; n) estágio da institucionalização dos serviços aos estudantes; o) melhores práticas compartilhadas; $p$ ) nível de autonomia dos atores do sistema UAB.

$\mathrm{Na}$ dimensão "o diagnóstico do sistema UAB" as variáveis estão organizadas em categorias, as saber: a) impactos no ensino superior; b) desafios do sistema UAB; c) influência dos fatores críticos pedagógicos nos resultados; d) influência dos fatores críticos administrativos nos resultados; e) influência dos fatores críticos financeiros nos resultados; f) influência dos fatores críticos docentes nos resultados; g) influência dos fatores críticos discentes nos resultados; $h$ ) influência dos fatores críticos dos polos nos resultados. As quatro dimensões de análise são consideradas imprescindíveis para avaliação da efetividade da UAB como política pública para EaD.

\section{CONSIDERAÇÕES FINAIS}

Ao longo do texto, sinalizamos indicadores que revelam a complexidade do sistema de 
educação superior brasileiro, destacando a participação da EaD como importante ferramenta para impulsionar a expansão da oferta. Neste sentido, um aspecto de grande importância refere-se à avaliação/regulação dessa oferta, não somente com a avaliação de cursos e instituições, mas principalmente pela avaliação da efetividade da política como um todo, através da avaliação do sistema. Assim, a partir dos resultados dessa avaliação pode-se verificar a efetividade da $U A B$, não somente na expansão das matrículas, como também na garantia da expansão com qualidade da educação superior. No que se refere à avaliação, visto que o importante é informar, trabalhar com as diferentes representações, interpretar as causalidades e pensar as estratégias de superação, é necessário que alguns fatores sejam levados em consideração como: a) repensar o papel da avaliação; b) valorizar a formação docente; c) Identificar os possíveis modelos de avaliação; d) a exigência da presencialidade; e) os altos índices de evasão; f) causas e consequências da evasão; g) formação de avaliadores; h) operacionalização das avaliações; i) identificação dos casos de sucesso; j) replicação de boas práticas.

Preliminarmente, os dados até aqui apresentados permitem-nos inferir que a EaD tem se tornado uma importante ferramenta para impulsionar a expansão da oferta do Ensino Superior no Brasil, porém que a busca de um modelo único talvez não seja a melhor solução para atender a diversidade brasileira. Pode-se então, optar pela adoção de parâmetros de qualidade, que permitam visibilidade e credibilidade aos cursos e/ou instituições credenciadas junto MEC, em detrimento da proposta atual de modelo de avaliação que prioriza aspectos técnicos. Surgindo como uma solução para garantir a expansão, mas a partir da consolidação de cursos e instituições e da garantia da qualidade na formação. Neste sentido, o presente artigo descreveu as dimensões e variáveis de análise a serem utilizadas para composição de um modelo de avaliação que identifique a efetividade do Sistema $U A B$ como política pública de $\mathrm{EaD}$, tornando possível, a partir da aplicação desse modelo como ferramenta de avaliação, identificar os potencialidades e deficiência do sistema.

\section{REFERÊNCIAS}

AAKER, D.A.; DAY, G.S. Marketing Research. Singapore: John Wiley e Sons, 1990.

BRASIL. Decreto no 5.800, de 8 de junho de 2006. Dispõe sobre o Sistema Universidade Aberta do Brasil - UAB. In: Diário Oficial da República Federativa do Brasil, Brasília, DF, 8 junho 2006a.

CAPES. Universidade Aberta do Brasil. In: Apresentação. Brasília: Fundação CAPES, 
2016a.

CAPES. Universidade Aberta do Brasil. In: Histórico. Brasília: Fundação CAPES, 2016b.

FERREIRA, M.; CARNEIRO, T. C. J. A institucionalização da educação a distância no ensino superior brasileiro: análise do Sistema UAB. In: Educação Unisinos, V. 19, №. 2. Canoas: Unisinos, maio/agosto, 2015. pp. 228-242.

FERRUGINI, L.; MORAIS, R.; PEREIRA, J. R.; SOUZA, D. L. Gestão das políticas públicas de educação a distância no Brasil: fragilidades e potencialidades. In: Anais dos $X$ ESUD - Congresso Brasileiro de Ensino Superior a Distância. Belém: UNIREDE, jun. 2013.

FREY, K. Políticas públicas: um debate conceitual e reflexões referentes à prática da análise de políticas públicas no Brasil. In: Planejamento e Políticas Públicas. Brasília: n. 21, p. 211-259, jun. 2000.

LIRA, L. A. R.; LIMA, B. F. Z.. Desafios da gestão de políticas públicas educacionais para formação de professores no Sistema UAB. In: EmRede. V.1, № 1. Porto Alegre: Unirede, jul. 2014. pp. 137-151.

MEC. Universidade Aberta do Brasil. In: Site do MEC. Brasília: MEC, 2016. Disponível em www.mec.gov.br/uab e acessado em 05/03/2017.

MELO, G. N.; SILVA, R. N. A gestão e a autonomia da escola nas novas propostas de políticas educativas para a América Latina. In: Estudos Avançados. V.12, № 5 . São Paulo: USP, 1991. pp. 45-60.

MERCADO, L. P. L.; PIMENTEL, F. S. C.; PINTO, I. M. B.; ARAUJO, M. S.; PINTO, A. C.. Indicadores da educação a distância no contexto da Universidade Aberta do Brasil: impactos acadêmicos, pedagógicos e sócio-econômicos. In: Revista EDaPECI Educação a Distância e Práticas Educativas Comunicacionais e Interculturais. Ano IV, Vol. 11, № 11. Maceio: UFAL, agosto 2012. pp. 1-20.

MICHELON, T.; RAZUCK, F. B.. O Sistema UAB: um estudo preliminar sobre a identificação dos fatores críticos da gestão integrada. In: EmRede. V. 3, № 2. Porto Alegre: UniRede, dez 2016. 
MILL, D.; CARMO, H.. Análise das dificuldades de educadores e gestores da educação a distância virtual no Brasil e em Portugal. In: SIED - Simpósio Internacional de Educação a Distância/EnPED - Encontro de Pesquisadores em Educação a Distância. São Carlos: UFSCar, 2012.

MILLER, D.C. Handbook of Research Design and Social Measurement. 3ª. Ed. New York, Longman, 1977.

NOGUEIRA, R. Elaboração e análise de questionários: uma revisão da literatura básica e a aplicação dos conceitos a um caso real. Rio de Janeiro: UFRJ/COPPEAD, 2002. 26p.

PERRIEN, J. Recherche en Marketing: méthodes et décisions. Gaetan Morin, Canada, 1986.

REUSE. Modelo de maturidade em colaboração (CollabMM). In: Equipe de reutilização de software. Rio de janeiro: UFRJ, 2013.

SARTURI, R. C.; WUNSCH, L.; TURCHIELO, L. B.; POMNITZ, N. C. UAB: desafios e possibilidades nas práticas docentes. In: Imagens da educação. V. 5, № 2. Santa Maria: UFSM, 2015. pp. 66-75.

TRIGUEIRO, M. G. S. O Ensino Superior Privado. Brasília: Paralelo 15, 2000.

UAB/UFES/IFES. Boas práticas de gestão de polos UAB - Espírito Santo (apresentação). Vitória: UFES/IFES, 2013.

ZUIN, A. A. S. Educação a distância ou educação distante? O programa UAB, o tutor e o professor virtual. In: Educação e Sociedade, Campinas, vol. 27, n. 96 - Especial, p. 935-954, out. 2006. 